\title{
SPECTRUM CHARACTERISTICS OF COTTON CANOPY INFECTED WITH VERTICILLIUM WILT AND INVERSION OF SEVERITY LEVEL
}

\author{
Bing Chen ${ }^{1}$, Keru Wang ${ }^{1,2}$, Shaokun Li $^{1,2,{ }^{*}}$, Jing Wang ${ }^{3}$, Junhua Bai ${ }^{1,2}$, \\ Chunhua Xiao ${ }^{1}$, Junchen Lai ${ }^{1}$ \\ ${ }^{1}$ Key Laboratory of Oasis Ecology Agriculture of Xinjiang Bingtuan, Shihezi University, \\ Research Center of Xinjiang Crop Yield. Shihezi University, shihezi 832000, xinjiang \\ ${ }^{2}$ Institute of Crop Science, Chinese Academy of Agricultural Sciences, The National Key \\ Facility for Crop Gene Research and Genetic Improvement, NFCRI, Beijing, 100081, \\ P. R. China \\ ${ }^{3}$ College of Resources and Environment, North west Science and Technology University of \\ Agriculture and Forestry, Yang ling, Shan xi 712100 \\ * Corresponding author, Address: P.O. Box 121, Chinese Academy of Agricultural Sciences \& \\ The National Key Facility for Crop Gene Research and Genetic Improvement NFCRI, \\ Institute of Crop Science, Beijing, 100081, P. R. China, Tel: +86-10-68918891, Email: \\ lishk@mail.caas.net.cn
}

Abstract: Verticillium wilt of cotton is one of the diseases of cotton with extensive occurrence and maximal harming in our country even in the world. Hyper spectrum remote sensing with the fine spectrum information has becoming the efficient method to monitor the verticillium wilt of cotton. The research was conducted in Xinjiang, the largest cotton plant region of China. The paper used data which was collected both canopy spectrum infected with verticillium wilt and SL (severity level) in the year 2005-2006, the quantitative correlation were analyzed between SL and canopy reflectance spectrum, derivative spectrum. The tested results indicated that spectrum characteristics of cotton canopy infected with verticillium wilt had better regularity with the increase of $\mathrm{SL}$ in different periods and varieties. Spectrum reflectance increased in visible light region $(620-700 \mathrm{~nm})$ with the increase of the SL, which inverted in nearinfrared region, and extreme signification in $780-1300 \mathrm{~nm}$. When SL got $25 \%$, cotton canopy infected with verticillium wilt could be used as a watershed and diagnosed index in an early time. The tested results also indicated there were evident characteristics of first derivative spectrum in these SL, it changed significantly in red edge ranges $(680-760 \mathrm{~nm})$ with different SL, red edge swing decreased, and red edge position equal moved to

Chen, B., Wang, K., Li, S., Wang, J., Bai, J., Xiao, C. and Lai, J., 2008, in IFIP International Federation for Information Processing, Volume 259; Computer and Computing Technologies in Agriculture, Vol. 2; Daoliang Li; (Boston: Springer), pp. 1169-1180. 
the blue. The results indicated that $1001-1110 \mathrm{~nm}$ and $1205-1320 \mathrm{~nm}$ were selected out as sensitive band regions to SL of canopy. These some inversion models for estimating cotton canopy infected with verticillium wilt all reached the most significantly level. At last, the results suggested that different spectrum characteristics of cotton canopy infected with verticillium wilt were obvious, the first derivative spectrum (FD $731 \mathrm{~nm}-\mathrm{FD}{ }_{1317 \mathrm{~nm}}$ ) will invert the cotton canopy SL accurately, and it may be used to forecast the position of cotton canopy infected with verticillium wilt in quantitatively.

Keywords: cotton; verticillium wilt; canopy spectrum; SL; inversion mode

\section{INTRODUCTION}

Verticillium wilt of cotton, which is widly- outbreak, strong- epidemic and highly- of outbreak, is one of the diseases of cotton with extensive occurrence and maximal harming in our country even in the world. Traditional monitoring method of verticillium wilt was that people investigated and sampled in field, which was limited time-consuming, hard-sledding, so prevention and cure of disease were effected to some extent. In this paper, spectrum characteristics of cotton canopy infected with verticillium wilt were analyzed, and inversion modes of SL were established. The studying results will provide theoretic support for further monitoring cotton verticillium wilt in large area by aviation and spaceflight remote sensing. As shown in paper (Huang et al. 2006; Ma et al. 2004), due to plant diseases and insect pests, some canopy spectrum parameters, such as LAI (leaf area index), biomass and coverage etc., had happened change, leading canopy spectrum reflectance correspondingly changed too, which made possibly fast monitoring occurrence and development of verticillium wilt of cotton in large area by remote sensing technology.

Some domestic and overseas scholars have had some correlate experiments on characteristics of canopy spectrum of plant diseases and insect pests, and made a great deal of achievements (Hamed Hamid Muhammed, 2005; Zhang et al. 2005; Bravo et al. 2003; Huang et al. 2003; $\mathrm{Wu}$ et al. 2002; Liu et al. 2002; Qiao et al. 2002; Chen, 2007). However, there have been few studies on surveying crop disease (especially to plant disease types) by remote sensing (Luo et al. 1997; Kefyalew Girma et al. 2005). When cotton was harmed by verticillium bacterium, the spectrum of cotton plant and remote sensing image will put up some especial diagnosis spectrum characteristics, which had different spectrum characteristics 
between single leaf and canopy, at present, however there are little report about them, it has become a unsubstantial technique on monitoring cotton growth situation by remote sensing. What are spectrum characteristics of cotton canopy infected with verticillium wilt? How to recognize cotton of disease and inverst SL of disease by remote sensing? So far, these studying have had little systemic reportion. Elucidating above mentioned questions, for disclosing spectrum mechanism of cotton canopy infected with verticillium wilt, monitoring district and trend of verticillium wilt, instructing reasonable distribution with breed resisted have very important meaning.

\section{MATERIALS AND METHODS}

\subsection{Design of experiments}

The experiment was conducted in cotton region of Shihezi in Xinjiang in the 2005-2006. The small area experiment was conducted in tentative cotton disease garden of institute of Key Laboratory of Oasis Ecology Agriculture in Shihezi university $\left(44^{\circ} 18^{\prime} \mathrm{N}, 86^{\circ} 03^{\prime} \mathrm{E}\right)$. The soil type was characterized as a soil-grey desert loam with organic matter content $1.93 \%$, alkalihydrolysis nitrogen $77.4 \mathrm{mg} \cdot \mathrm{kg}^{-1}$, rapidly available phosphorus $93 \mathrm{mg} \cdot \mathrm{kg}^{-1}$ and rapidly available potassium $315 \mathrm{mg} \cdot \mathrm{kg}^{-1}$ in $20 \mathrm{~cm}$ depth, former stubble plant was cotton. The selected cultivars in this experiment were some cotton varieties with different plant types and same growing periods, for example, Xin Luza 7(XLZ-7), Xin Luza 8(XLZ-8), Xin Luza 13(XLZ-13), Xin Luza 24(XLZ-24), and Zhong Mian 36(ZM-36). The plot area was $42.5 \mathrm{~m}^{2}$ with three repeats, the plant density was 240000 plants per ha, and row spacing was $60 \mathrm{~cm}+30 \mathrm{~cm}$, the seeding time was the $20 \mathrm{rd}$ April, plant mode was onfilm order programmer with under-film drip irrigation. Irrigation carried out under-film irrigation with $3300 \mathrm{~m}^{3} \cdot \mathrm{hm}^{2}$, fertilizer amounts were pure nitrogen $300 \mathrm{~kg} \bullet \mathrm{ha}^{-1}, 150 \mathrm{~kg} \bullet \mathrm{ha}^{-1} \mathrm{P}_{2} \mathrm{O}_{5}, 75 \mathrm{~kg} \bullet \mathrm{ha}^{-1} \mathrm{~K}_{2} \mathrm{O}$. Besides, the plot had not weed, the other treatments were applied according to high yield cultivation mode in the local region. The field area experiment was conducted in cotton region of Shihezi, locating 19 company at 143 regiment, seed station at 147 regiment and 11 company at 148 regiment, where all had occurred continuously verticillium wilt in large area in the past years. Cultivars in this experiment were the same as small area experiment. Besides; some new cotton cultivars were selected, for example 602 and 4432. The soil type was characterized as 
a soil-grey desert loam with middle fertility, and the seeding time date was on the last ten-day of April, other was the same as small area experiment.

\subsection{DI (Disease index) investigation and SL classification}

DI was investigated with five point method, which was, symmetrical five points were selected in treatment plot to investigate DI, and ten cotton plants (about $1 \mathrm{~m}^{2}$ ) were selected out in very one point after DI was investigated. SL of single plant was divided into five grades, namely, normal single plant was 0 grade; numbers of leave with disease on single plant had not exceed $25 \%$ as 1 grade; numbers of leave with disease on single plant between $25 \%$ and $50 \%$ as 2 grade; numbers of leave with disease on single plant had exceed $50 \%$ as 3 grade; single plant had died or almost died as 4 grade. After finishing investigating, single plant numbers and grade were registered in every point, and calculated formula of DI with tested canopy as follows (Zhang et al. 2005):

$$
D I=\frac{\sum(\mathrm{X} * f) \times 100}{n * \sum f}
$$

In the formula, $x$ denoted grade value from every grade; $n$ denoted grade number from highest grade; $f$ denoted plant numbers from every grade. Then, according to different DI with tested canopy, SL of tested canopy were divided into five grades, namely, normal (b0): $\mathrm{DI}=0$; mild degree (b1): DI was between 0 and 25; moderate degree (b2): DI was between 25 and 50; severe degree (b3): DI was between 50 and 75; most severity degree (b4): DI was between 75 and100.

\subsection{Hyper spectrum reflectance data collections}

Hyper spectrum reflectance measurements were taken under clear sky conditions from 11:30 to 14:00 (Beijing local time) using an ASD Field spec Pro FR 2500 spectrometer (Analytical Spectral Devices, Boulder, CO, USA) fitted with a 512 spectrum bands, operating in the $350-2500 \mathrm{~nm}$ spectrum region with a sampling interval of $1.4 \mathrm{~nm}$ between 350 and $1000 \mathrm{~nm}$, and $2 \mathrm{~nm}$ between 1000 and $2500 \mathrm{~nm}$, and with spectrum resolution of $3 \mathrm{~nm}$ 
between 350 and $1000 \mathrm{~nm}, 10 \mathrm{~nm}$ between 1000 and $2500 \mathrm{~nm}$. A panel radiance measurement was taken with $25^{\circ}$ field of view before and after the canopy measurement by two scans each time, and scans time of each sample was $0.2 \mathrm{~s}$, sample of spectrum reflectance was measured five times in every plot, finally, spectrum reflectance value of each plot was acquired by averaging. Different SL of cotton canopy infected with verticillium wilt was measured by the method that the sensor probe of the spectrometer was taken vertically from a height of $1.5 \mathrm{~m}$ above canopy, as a standard, the solar radiation spectrum value was calibrated by using the standard white board $\left(40 \mathrm{~cm} * 40 \mathrm{cn}, \mathrm{BaSO}_{4}\right)$ before every measurement canopy of spectrum. In the year 2005-2006, cotton canopy infected with verticillium wilt was measured in different growth stages, for example, peak budding period (20 June), peak flowering period (19 July, 21 July), peak bolling period (3 August, 12 August), peak opening period (4 September).

\subsection{Data analysis methods}

Using view spec program software (made ASD company) managed primitive canopy reflectance spectrum data, we obtained reflectance spectrum data and reflectance curve, at the same time, the first derivative spectrum method was selected with matlab 7.01 software, and calculated formula of the first derivative spectrum as follows (wang et al., 2005):

$$
\rho^{\prime}\left(\lambda_{i}\right)=\frac{\left[\rho\left(\lambda_{i+1}\right)-\rho\left(\lambda_{i-1}\right)\right]}{2 \Delta \lambda}
$$

In the formula, $\lambda_{i}$ denoted wave length value from ${ }_{i}$ wave band; $\rho\left(\lambda_{i}\right)$ denoted spectrum value from $\lambda_{i}$ wave length; $\Delta \lambda$ denoted space from $\lambda_{i-1}$ to $\lambda$, which was decided by sampling interval of spectrum. The correlation between original spectrum, first derivative spectrum and SL was analyzed by Excel 2003, wave band with spectrum characteristic and combination parameters from wave band were extracted from them (Huang et al. 2003; $\mathrm{Gu}, 2003)$. On the other hand, SPSS 10.0 software was applied in statistics analysis, number of samples used in establishing model and testing were 89 and 73 respectively. 


\section{RESULTS AND ANALYSES}

\subsection{Total spectrum characteristics on cotton canopy infected with verticillium wilt}

The results indicated that spectrum reflectance of cotton canopy had obvious diversity between normal cotton and disease cotton with verticillium wilt (Figure 1,2). The value of spectrum reflectance of cotton canopy infected with verticillium wilt was higher than normal in visible light wave band, and it had little influence by cotton growing periods. The value was lower than normal canopy in near-infrared wave band, and it had little influence by cotton growing periods and varieties.

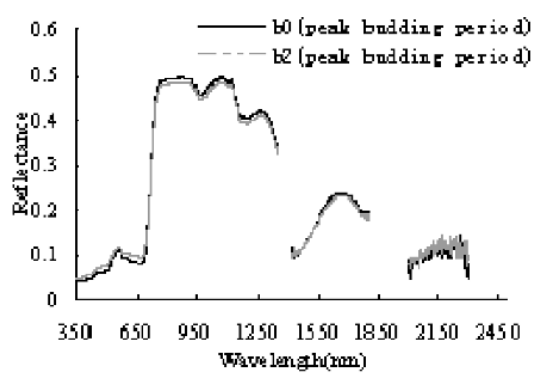

a

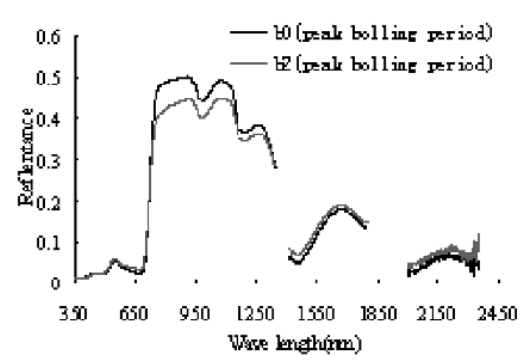

c

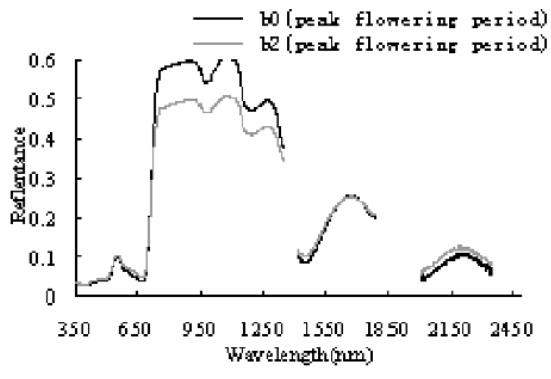

$\mathrm{b}$

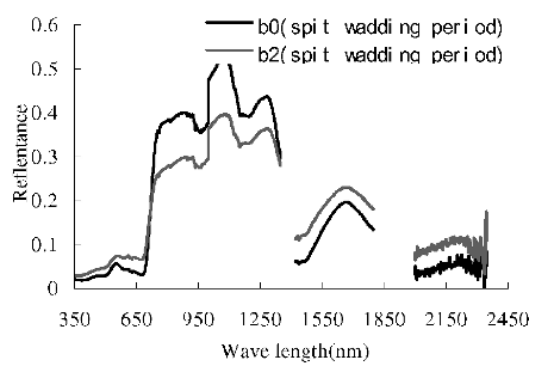

d

Fig. 1. Spectrum graph between the disease and CK treatment at different growth stages of cotton (XLZ-7)

\subsection{Spectrum characteristics of cotton canopy verticillium wilt with different $\mathrm{SL}$}

The results indicated from analyzing canopy spectrum of different disease degrees (Figure 3), spectrum reflectance raised in visible light region (620- 
$700 \mathrm{~nm}$ ) with the increase of the SL, however, it inverted in near-infrared region, spectrum reflectance fallen down with the increase of the SL, and extreme signification in 780-1300 nm namely, spectrum reflectance of normal canopy (b0) was the highest in near-infrared region, mild degree canopy (b1) took second place, most severity degree canopy (b4) was the lowest. Canopy spectrum from different varieties and different growth stages had homologous change trend, but spectrum reflectance value was different. Consequently, spectrum characteristics difference of cotton canopy with different SL can be used to recognize SL of verticillium wilt.

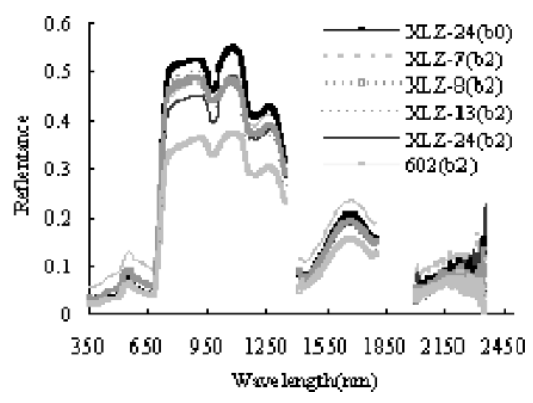

Fig. 2. The reflectance spectrum curve of varieties cotton canopy of verticillium wilt in peak bolling stage

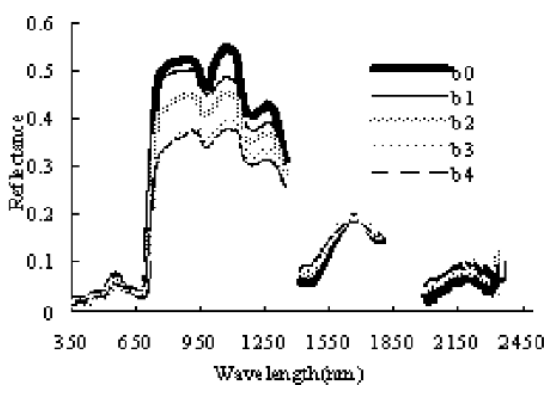

Fig. 3. The reflectance spectrum curve of cotton canopy of verticillium wilts with different SL

In near-infrared wave band (780-1300 nm), discrepancy of canopy spectrum reflectance with different SL was obvious between diseases and normal (Figure 4). With the SL increasing, discrepancy of canopy spectrum reflectance was increased generally, when SL got b1, canopy reflectance of diseases was lower by 3\% than normal, but when SL got b2 (25\%), it lower $8 \%$ than normal. So b2 $(25 \%)$ could be used as a watershed and diagnosed index in early time.

This study indicated (Figure 5), wave bands, which located the red edge region in first derivative spectrum, changed biggest, namely, maximum of 
the first derivative spectrum and corresponding wavelength between $680 \mathrm{~nm}$ and $760 \mathrm{~nm}$. As for normal canopy, the red edge of cotton canopy infected with verticillium wilt with different SL all had "double swings" phenomenon, and "double swings" descended at the same time, besides, red edge position all moved to the short-wave direction obviously, namely, red edge swing decreased, and red edge position happened "blue movement".

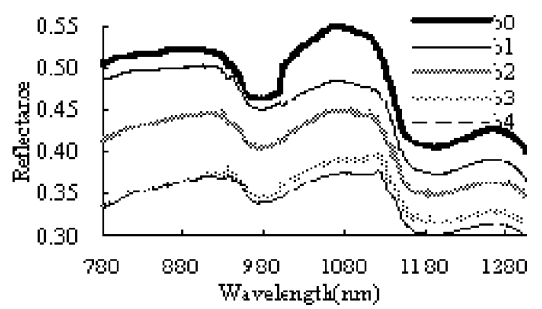

Fig. 4. The spectrum curve of cotton canopy of verticillium wilts with different SL in 780$1300 \mathrm{~nm}$

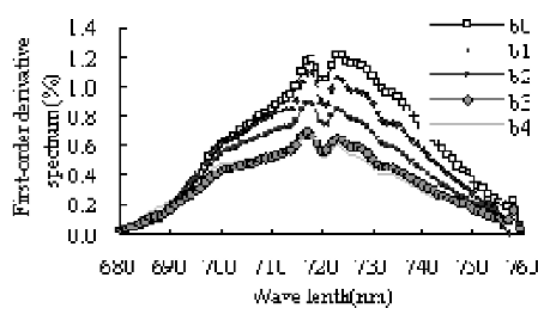

Fig. 5. The red edge of cotton canopy of verticillium wilts with different SL

\subsection{Selecting sensitive wave band on cotton canopy infected with verticillium wilt and SL invertion}

The spectrum reflectance curve shape of cotton canopy which infected with verticillium wilt with different SL was likeness. However, the depth value of band swing and valley was different; namely, cotton canopy spectrum infected with verticillium wilt had certain regular (Figure 3). Except for effect on atmosphere and water, the sensitive wave bands were selected in 400-1350 nm, 1400-1800 $\mathrm{nm}$ and 1950-2350 nm. After the correlation between SL of cotton infected with verticillium wilt and the canopy spectrum reflectance was analyzed, we got correlation coefficient curve (Figure 6). The result indicated, the correlation between spectrum reflectance and SL was positive in 620-700 $\mathrm{nm}$ (visible light region), and was negative in 780-1300 nm (near-infrared area), which showed consistent with spectrum 
reflectance, the spectrum reflectance rose in visible light region, and descended in near-infrared region with SL aggravation, and the Correlation between spectrum reflectance and SL had arrived at the best significantly level in 733-1350 nm bands, so they were selected out as sensitive band region to canopy SL of verticillium wilt, and 1001-1110 nm and 1205-1320 $\mathrm{nm}$ were selected out as spectrum sensitive wave band of them. Due to the correlations between spectrum reflectance and SL was maximum at $806 \mathrm{~nm}$ and higher at $690 \mathrm{~nm}, 1455 \mathrm{~nm}$, they were selected out, and base on their combinations, estimation models of canopy infected with verticillium wilt with SL were established. At the same time, estimation models were tested. As shown (Table 1), the wave band combinations model, which was established by R806 nm - R1455 nm, had higher estimative accuracy, the RMES was the least (0.743).

Table 1. The inversing model and test for colony severity level of cotton with verticillium wilt

\begin{tabular}{lcccc}
\hline Wave Band Parameter $(\lambda)$ & Diagnosing model $(\mathrm{sl})$ & $\begin{array}{c}\text { Determination } \\
\text { coefficients } \\
\left(\mathrm{R}^{2}\right)\end{array}$ & $\begin{array}{c}\text { Root mean } \\
\text { square error } \\
(\mathrm{RMES})\end{array}$ & $\begin{array}{c}\text { Correlative } \\
\text { coefficients } \\
(\mathrm{r})\end{array}$ \\
\hline $\mathrm{R}_{806 \mathrm{~nm}}$ & $\mathrm{sl}=-11.64 \mathrm{x}+7.0722$ & $0.675^{* *}$ & 0.799 & $0.798^{* *}$ \\
$\mathrm{R}_{806 \mathrm{~nm}}-\mathrm{R}_{690 \mathrm{n}}$ & $\mathrm{sl}=-11.66 \mathrm{x}+7.0722$ & $0.675^{* *}$ & 0.805 & $0.789 * *$ \\
$\mathrm{R}_{806 \mathrm{~nm}}-\mathrm{R}_{1455 \mathrm{~nm}}$ & $\mathrm{sl}=-10.588 \mathrm{x}+5.6764$ & $0.690^{* *}$ & 0.743 & $0.824^{* *}$ \\
$\mathrm{R}_{806 \mathrm{~nm}} / \mathrm{R}_{1455 \mathrm{~nm}}$ & $\mathrm{sl}=-0.5309 \mathrm{x}+4.7848$ & $0.558^{* *}$ & 0.910 & $0.733^{* *}$ \\
$\left(\mathrm{R}_{806 \mathrm{~nm}}-\mathrm{R}_{690 \mathrm{~nm}}\right) /\left(\mathrm{R}_{806 \mathrm{~nm}}+\mathrm{R}_{690 \mathrm{~nm}}\right)$ & $\mathrm{sl}=-8.5564 \mathrm{x}+7.4851$ & $0.537 * *$ & 0.901 & $0.747 * *$ \\
$\mathrm{FD}_{731 \mathrm{~nm}}$ & $\mathrm{sl}=-509.42 \mathrm{x}+5.3279$ & $0.715^{* *}$ & 0.750 & $0.846^{* *}$ \\
$\mathrm{FD}_{1317 \mathrm{~nm}}$ & $\mathrm{sl}=2184.4 \mathrm{x}+5.4427$ & $0.627 * *$ & 0.804 & $0.818^{* *}$ \\
$\mathrm{FD}_{731 \mathrm{~nm}}-\mathrm{FD}_{589 \mathrm{~nm}}$ & $\mathrm{sl}=-484.88 \mathrm{x}+5.2745$ & $0.713^{* *}$ & 0.753 & $0.844 * *$ \\
$\mathrm{FD}_{731 \mathrm{~nm}}-\mathrm{FD}_{1317 \mathrm{~nm}}$ & $\mathrm{sl}=-438.41 \mathrm{x}+5.5722$ & $0.741^{* *}$ & 0.730 & $0.853^{*}$ \\
\hline
\end{tabular}

Note: significance at the $P>0.01$ confidence interval. Number of samples used in establishing model and testing are 89 and 73 respectively.

After the characteristics of first derivative spectrum on cotton canopy infected with verticillium wilt were analyzed, the correlation between SL of disease and the first derivative spectrum was present (Figure 7). Because the correlation between first derivative spectrum and SL was higher at $589 \mathrm{~nm}$, $731 \mathrm{~nm}$ and $1317 \mathrm{~nm}$, they were selected out and base on their combinations, estimation models of canopy infected with verticillium wilt with SL were established, at the same time, those models were tested. As shown (Table 1), the wave band combinations model, which was established by FD731 nm - FD1317 nm, showed higher estimate accuracy, the RMES was the least (0.735). Therefore, we drown an conclusion that using first derivative spectrum, SL of cotton canopy infected with verticillium wilt could be estimated. Moreover, the testing result of model indicated, the $b$ value was not zero in regression model, and possible reason was that 


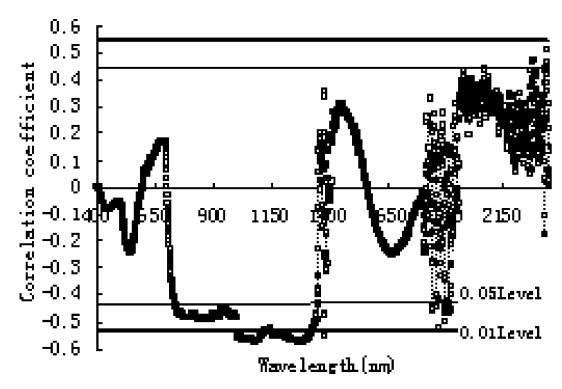

Fig. 6. Correlation between the cotton canopy of verticillium wilt different SL and spectrum reflectance

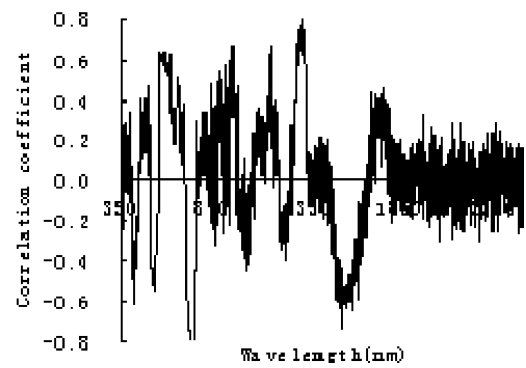

Fig. 7. The correlation analyses between the first derivatative spectrum datas and SL

establishing and testing model samples were come from different years and varieties, and then certain system error was inevitable.

\section{CONCLUSIONS AND FUTURE WORKS}

From the above analysis, the main results can be described as follows:

(1) Spectrum characteristic on cotton canopy infected with verticillium wilt had better regularity with the increase of SL in different periods and varieties. With the aggravation of the SL, spectrum reflectance rose at visible light region (620-700 $\mathrm{nm}$ ), and declined at near-infrared region, and however, it was special signification at 780-1300 nm.

(2) When SL got 25\%, cotton canopy infected with verticillium wilt could be used as a watershed and diagnosed index in early time.

(3) The characteristics of first derivative spectrum on verticillium wilt indicated, wave bands, which located red side district $(680-760 \mathrm{~nm})$ changed significantly, from normal to mild to most severity disease, red edge swing decreased, while the red edge position equal moved to the blue, presented special spectrum characteristics of disease.

(4) The results indicated that $1001-1110 \mathrm{~nm}$ and $1205-1320 \mathrm{~nm}$ can be selected out as sensitive bands region to monitor SL of canopy. Base on above study, some inversion models, which estimated canopy infected with verticillium wilt, were established, and those models in which the first derivative spectra at $\mathrm{FD}_{731 \mathrm{~mm}}-\mathrm{FD}_{1317 \mathrm{~mm}}$ would improve accuracy the SL of cotton canopy, and it may be acted as the best recognized model to SL of verticillium wilt. 
(5) Future work is analyses various stresses, including disease bring diversities on physiology, bio-chemical mechanism in cotton and its respond with canopy parameters of verticillium wilt on the spectrum, establish higher precision spectrum identification models and canopy parameters inversion modes.

\section{ACKNOWLEDGEMENTS}

This work was supported by National High Technology Research and Development Program of China (863 programmes 2006AA103A302, 2006AA10Z207) and Key Laboratory of Oasis Ecology Agriculture of Xinjiang Bingtuan open task.

\section{REFERENCES}

Bravo, C, Moshou, D, West, J McCartney, A. 2003, Ramon H. Early disease detection in wheat fields using spectral reflectance. Biosystems Engineering, 84, 137-145.

Chen Pengcheng. 2006, Application of groung-based hyperspectral remote sensing to detect spider mite. (In Partial Fulfillment of the Requirements for the Degree of Postgraduation), Xinjiang, Shi hezi university, 14-15 (in Chinese with English abstract)

Guo Ni. 2003, Vegetation index and its advances. Arid Meteorology, 21, 71-75 (in Chinese with English abstract)

Hamed Hamid Muhammed. 2005, Hyperspectral crop reflectance data for characterizing and estimating fungal disease severity in wheat. Biosystems Engineering, 91, 9-20.

Huang Lin, Zhang Xiaoli, Shi Ren. 2006. Current status and problems in monitoring forest damage caused by diseases and insects based on remote sensing. Remote sensing information, 71-75 (in Chinese with English abstract)

Huang muyi, Wang jihua, Huang wenjiang, Huang yide, Zhao chunjiang, Wan anmin. 2003, Hyperspectral character of stripe rust on winter wheat and monitoring by remote sensing. Transactiongs of The CSAS, 19, 154-158 (in Chinese with English abstract)

Kefyalew Girma, Mosali, J, Raun, W R, Freeman, K W, Freeman, K.W, Martin, L.K, Solie, J.B, Stone, M L I, 2005, Dentification of optical spectral signatures for detecting cheat and ryegrass in winter wheat. Crop Science, 45, 477-485.

Liu Liangyun, Huang Muyi, Huang Wenjiang, Wang Jihua, Zhao Chunjiang, Zheng Lanfen, Tong Qingxi. 2004, Monitoring stripe rust disease of winter wheat using multi-temporal hypersoectral airborne data. Journal of Remote Sensing, 8, 276-281 (in Chinese with English abstract)

Luo Xiuling, Zhou Hesheng, Xue Qin, Zhang Changhong, Wang Zhigang, Zhou Xu. 1997, Satellite multiangular remote sensing for distinguishing areas harmed by mice and insects in the grassland. Journal of remote sensing, supple, 1, 212-219 (in Chinese with English abstract) 
Ma Jianwen, Han Xiuzhen, Ha Sibagan, Wang Zhigang, Yan Shouxun, Dai Qin. 2004, Remote sensing new model for monitoring the east Asina migratory locust infections based on its breeding circle. Journal of remotes sensing, 8, 370-377 (in Chinese with English abstract)

Qiao Hongbo, Jian Guiliang, Zhou Yafei, Cheng Dengfa. 2007, Influence of Fusavium wilt to differenct resistance cultivars on spectrum of cotton. Cotton science, 19, 155-158 (in Chinese with English abstract)

Wang Xiuzhen, Li Jianlong, Tang Yanlin. 2004, Approach the action of derivative spectral for determining agronomic parameters of cotton. Journal of Huanan Agriculture University, 25, 17-21 (in Chinese with English abstract)

Wu Suwen, Wang Renchao, Chen Xiaobin, Shen zhangquan, shi zou. 2002, Effect of rice leaf blast on spectrum reflectance of rice. Journal Of Shanghai Jiaotong University, 20, 73-84 (in Chinese with English abstract)

Zhang Kaiqiang, Li Sshengjian, Lu Shenbing, Luo Hua, Wu Yinghua, Li Xiaobing, Wu Anping, Qing Jiugang. 2004. Fusarium and verticillium wilt of cotton were investigated in plain of jianghan in 2004. Cina cotton, 31, 38-39 (in Chinese)

Zhang Minghua, Qin Zhihao, Liu Xue. 2005, Remote sensed spectral imagery to detect late blight in field tomatoes. Precision Agriculture, 6, 489-508. 